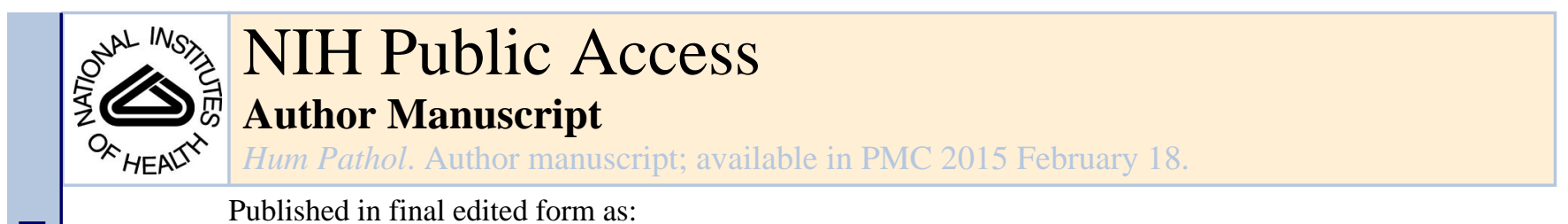

Published in final edited form as:

Hum Pathol. 2014 June ; 45(6): 1130-1138. doi:10.1016/j.humpath.2014.01.013.

\title{
Clear cell papillary renal cell carcinoma: micro-RNA expression profiling and comparison with clear cell renal cell carcinoma and papillary renal cell carcinoma ${ }^{\star}$
}

\author{
Enrico Munari, MD ${ }^{\mathrm{a}, 1}$, Luigi Marchionni, MD, $\mathrm{PhD}^{\mathrm{b}, 1}$, Apurva Chitre, $\mathrm{BS}^{\mathrm{b}}{ }^{\text {, Masamichi }}$ \\ Hayashi, MDc, Guido Martignoni, MD ${ }^{\mathrm{d}, \mathrm{e}}$, Matteo Brunelli, MD, PhD ${ }^{\mathrm{d}}$, Stefano Gobbo, MD ${ }^{\mathrm{d}, \mathrm{e}}$, \\ Pedram Argani, MDa, Mohamad Allaf, MD ${ }^{f}$, Mohammad O. Hoque, PhDc, ${ }^{\star}$, and George J. \\ Netto, MDa,b,f, ${ }^{a}$ \\ aDepartment of Pathology, Johns Hopkins University, Baltimore, MD 21287, USA \\ bDepartment of Oncology, Johns Hopkins University, Baltimore, MD 21287, USA \\ 'Department of Otolaryngology, Johns Hopkins University, Baltimore, MD 21287, USA \\ dDepartment of Pathology and Diagnostics, University of Verona, Verona 37134, Italy \\ eAnatomic Pathology, Pederzoli Hospital, Peschiera d/G, Verona 37019, Italy \\ fDepartment of Urology, Johns Hopkins University, Baltimore, MD 21287, USA
}

\section{Summary}

Clear cell papillary renal cell carcinoma (CCPRCC) is a low-grade renal neoplasm with morphological characteristics mimicking both clear cell renal cell carcinoma (CCRCC) and papillary renal cell carcinoma (PRCC). However, despite some overlapping features, their morphological, immunohistochemical, and molecular profiles are distinct. Micro-RNAs (miRNAs) are small noncoding RNAs that play a crucial role in regulating gene expression and are involved in various biological processes, including cancer development. To better understand the biology of this tumor, we aimed to analyze the miRNA expression profile of a set of CCPRCC using microarray and quantitative reverse transcription-polymerase chain reaction. A total of 15 cases diagnosed as CCPRCC were used in this study. Among the most differentially expressed miRNA in CCPRCC, we found miR-210, miR-122, miR-34a, miR-21, miR-34b*, and miR-489 to be up-regulated, whereas miR-4284, miR-1202, miR-135a, miR-1973, and miR-204 were downregulated compared with normal renal parenchyma. To identify consensus of differentially regulated miRNA between CCPRCC, CCRCC, and PRCC, we additionally determined differential miRNA expression using 2 publically available microarray data sets from the NCBI Gene

\footnotetext{
Disclosure/Conflict of Interest: The authors declare no conflict of interest.

(C) 2014 Elsevier Inc. All rights reserved.

"Corresponding authors: M.O. Hoque is to be contacted at Department of Otolaryngology, Johns Hopkins University, 1550 Orleans St, CRB II, 5th Floor, 21231 Baltimore, MD, USA. G.J. Netto, Department of Pathology, Urology and Oncology, Johns Hopkins University, 401 N. Broadway, 2242 Weinberg, 21231 Baltimore, MD, USA. mhoque1@jhmi.edu (M. O. Hoque), gnetto1@jhmi.edu (G. J. Netto).

${ }^{1}$ Enrico Munari and Luigi Marchionni contributed equally to the study.

Supplementary data

Supplementary data to this article can be found online at http://dx.doi.org/10.1016/j.humpath.2014.01.013.
} 
Expression Omnibus database (GSE41282 and GSE3798). This comparison revealed that the miRNA expression profile of CCPRCC shows some overlapping characteristics between CCRCC and PRCC. Moreover, CCPRCC lacks dysregulation of important miRNAs typically associated with aggressive behavior. In summary, we describe the miRNA expression profile of a relatively infrequent type of renal cancer. Our results may help in understanding the molecular underpinning of this newly recognized entity.

\section{Keywords}

Clear cell papillary renal cell carcinoma; Micro-RNA; Profiling; Renal cancer

\section{Introduction}

Micro-RNAs (miRNAs) are small noncoding RNAs that play a crucial role in regulating gene expression and are involved in various biological processes, including cancer development [1]. Several studies investigating miRNA expression in renal cell carcinoma (RCC) highlighted the importance of these small RNAs in the development of this disease $[2,3]$. miRNA panels predicting recurrence and unfavorable prognosis have been identified; furthermore, different miRNAs have also been shown to accurately discriminate between the most common types of renal tumors [4]. Clear cell RCC (CCRCC) and papillary RCC (PRCC) account for up to 90\% of all RCCs [5] and show distinct morphological, immunohistochemical, and molecular features. CCRCC is characterized by cells with clear cytoplasm, expression of carbonic anhydrase IX (CAIX) and CD10, alteration of the VHL gene, and other recurrent copy number alterations including $5 q$ gains and $9 p$ loss [6-8]. PRCC, on the other hand, shows papillary architecture, expression of cytokeratin 7 (CK7), $a$-methylacyl-CoA racemase (AMACR), and trisomies of chromosomes 7 and 17 [5]. Advanced CCRCC and PRCC are aggressive tumors with poor prognosis.

Since the publication of the 2004 World Health Organization classification of genitourinary tumors [5], new entities have been described, including renal angiomyoadenomatous tumor, RCC with prominent leiomyomatous proliferation, and clear cell papillary renal cell carcinoma (CCPRCC). CCPRCC is a renal epithelial neoplasm originally described in kidneys with end-stage renal disease and subsequently found outside this setting $[9,10]$. CCPRCC is a low-grade renal neoplasm with morphological characteristics mimicking both CCRCC and PRCC. However, despite some overlapping features, their morphological, immunohistochemical, and molecular profiles are distinct. These tumors show a unique immunoprofile characterized by the expression of CK7 and CAIX as well as lack of staining for AMACR [10-12]. Molecular genetic changes in CCPRCC are also distinct, as alterations of chromosome 3 and the $V H L$ gene and copy number gains of chromosomes 7 and 17 are lacking [10,12].

In this study, we aimed to analyze the miRNA expression profile of a set of CCPRCC and evaluated similarities and differences with CCRCC and PRCC to better understand the biologic characteristic of this newly recognized entity. 


\section{Materials and methods}

\subsection{Case selection}

A total of 15 cases diagnosed as CCPRCC over the years 2003 to 2013 were retrieved from the archives of The Johns Hopkins University Hospital and the University of Verona. The institutional review board of both institutions approved this study. Demographic and clinical data were collected.

All available hematoxylin and eosin slides were reviewed, and diagnosis was confirmed based on the presence of morphological and immunohistochemical features previously reported by others [9-11]. These included morphological features as the presence of papillary architecture, diffuse cytoplasmic clarity, and typical arrangement of nuclei away from the basement membrane. In all cases, the diagnosis of CCPRCC was supported by the typical immunohistochemical profile characterized by expression of CAIX and CK7 and negativity for AMACR, along with variable expression of CD10. Tumors were assessed for size, pathologic stage, Fuhrman nuclear grade, laterality, and presence of associated endstage renal disease. Of the 15 samples included in the current study, 4 were also previously assessed for $V H L$ mutation, $V H L$ methylation, chromosome 3p, and whole genomic status [13].

\subsection{RNA extraction}

In each case, a representative formalin-fixed, paraffin-embedded tissue sample was chosen for RNA extraction. Ten 8- $\mu$ m-thick unstained slides were cut from blocks, and a sterile scalpel was used to microdissect tumor cells ( $280 \%$ ) after deparaffinization with xylene and ethanol washing. Total RNA was extracted using the RecoverAll Total Nucleic Acid Isolation (Ambion, Austin, TX) according to manufacturer's protocol. RNA quantity was measured by using NanoDrop Spectrophotometer ND-1000 (Thermo Scientific, Waltham, MA). RNA quality was evaluated using Bioanalyzer 2100 (Agilent Technologies, Santa Clara, CA).

\subsection{Microarray hybridization}

Four matched tumor and normal samples constituted our discovery set and were submitted for microarray analysis. This was performed using the Human miRNA Microarray Kit Release 16.0, 8×60K (G4870A; Agilent Technologies) according to manufacturer's protocols. The array contains 1205 human miRNAs from the Sanger database v16.0 (http:// microrna.sanger.ac.uk/sequences/). Only samples with $28 \mathrm{~S} / 18 \mathrm{~S}>1.2$, RIN $>8$, and detectable miRNA were used for the analysis.

Microarray hybridization was performed at Sidney Kimmel Cancer Center Microarray Core Facility at Johns Hopkins University using manufacturer's instruction, as previously described [14]. Data were acquired with Agilent Feature Extraction 9.5.3.1 software for miRNA microarray. Two data files are generated for each array: Feature Extraction file contains signal intensities from all individual probes and GeneView file contains summarized signal intensities for each miRNA by combining intensities of replicate probes and background subtraction. 


\subsection{Technical and independent set validation of microarray data}

Individual TaqMan microRNA assays (Applied Biosystems, Austin, TX) were used for technical validation of microarray findings in the above discovery set of 4 cases as well as 11 additional cases (independent validations set). The individual miRNAs were selected among the miRNAs that showed to be the most differentially expressed in the microarray experiments. RNU6B was used as reference gene for normalization. Total RNA (10 ng) was reverse transcribed using TaqMan MicroRNA Reverse Transcription Kit (Applied Biosystems). Quantitative reverse transcription-polymerase chain reaction (qRT-PCR) was performed using TaqMan Universal Master Mix II (Applied Biosystems) on a 7900HT Fast Real Time PCR System according to the manufacturer's protocol. All samples were run in triplicate. Normalized signal levels for each miRNA were calculated using comparative cycle threshold method ( $\Delta \Delta \mathrm{CT}$ method) [15].

\subsection{Statistical analysis}

miRNA expression data were processed for statistical analysis using packages from R/ Bioconductor (www.bioconductor.org), as previously described [16-18]. Briefly, a generalized linear model was fit for each miRNA to estimate expression differences between clear cell papillary tumor and normal kidney, and an empirical Bayesian approach was used to moderate SEs of expression fold change. Multiple testing corrections were performed using the Benjamini-Hochberg method. To identify consensus of differentially regulated miRNA between renal carcinoma histologic types, we additionally determined differential miRNA expression using 2 publically available microarray data sets from the NCBI Gene Expression Omnibus database (GSE41282 and GSE3798). In these 2 data sets, we identified the miRNA expression profiles that distinguish the different tumor groups from normal counterparts. The meta-analysis was performed by comparing the lists of differentially expressed miRNA between the groups of interest independently obtained from each analyzed data set with a false discovery rate of $10 \%$ or less. We also performed comparisons between pairs of data sets using Correspondence At the Top (CAT) plots. CAT plot confidence intervals were based on the hypergeometric distribution, as previously described [18].

\section{Results}

\subsection{Clinicopathological characteristics and immunohistochemical profile}

Clinicopathological characteristics of tumors are summarized in Table 1.

There were 9 females and 6 males, and the mean age was 61 years (range, 36-70 years). The surgical treatment was partial nephrectomy in 13 patients and total nephrectomy in 2 patients. One patient had end-stage renal disease (ESRD). Mean diameter of tumors was 3 $\mathrm{cm} ; 12$ tumors were pT1a, whereas the remaining 3 were pT1b. Microscopically, all tumors showed the typical nuclear arrangement away from the basement membrane and were Fuhrman nuclear grade 2. We did not encounter necrosis or sarcomatoid transformation in any of our cases. 
Typical morphological and immunohistochemical characteristics of the tumors are depicted in Fig. 1.

Immunohistochemically, all tumors were positive for CK7 and CAIX; AMACR and CD10 were available in a subset of cases (14 and 12 cases, respectively); AMACR stain was negative in $14 / 14$ cases $(100 \%)$, whereas CD10 showed a variable degree of positivity (5/12 cases, $42 \%)$.

\subsection{Genome-wide miRNA expression profiling of CCPRCC}

Among the 1205 miRNAs contained in the microarray, 342 mature miRNA were differentially expressed between CCPRCC and normal samples with a false discovery rate (FDR) of less than $10 \%$ (Supplementary Table S1). Table 2 shows the most significantly differentially expressed (FDR $<5 \%$ ) miRNAs in CCPRCC compared with matched normal renal parenchyma.

To identify differentially expressed miRNAs between CCPRCC, CCRCC, and PRCC, we analyzed the GSE41282 data set, containing the expression profile of 6 CCRCC and 14 PRCC samples with corresponding normal tissue to derive miRNA gene expression profiles associated with CCRCC and PRCC compared with normal kidney. We respectively identified 10 and 22 miRNA moieties as differentially expressed (Supplementary Table S2a and S2b). We further analyzed the GSE37989 data set, containing microarray-based profiles of 9 metastatic CCRCCs and 12 primary CCRCCs with corresponding nonmalignant tissue to identify the miRNA profiles associated with primary and metastatic CCRCC [21]; we identified 87 and 174 differentially expressed miRNAs, respectively (Supplementary Table S3a and S3b). We finally compared our data from CCPRCC to the above data sets. We performed this cross-platform comparison using the most differentially expressed miRNA. In particular, we compared gene expression profiles distinguishing CCPRCC, CCRCC, and PRCC from normal samples by CAT plots using the results from our profiling and the analysis of the GSE41282 data set. This comparison revealed that a number of up-regulated miRNAs in CCPRCC are highly similar to those up-regulated in CCRCC, whereas downregulated miRNAs in CCPRCC are similar to those down-regulated in PRCC (Fig. 2A). We used a similar approach to compare our CCPRCC findings with miRNA profiles of primary and metastatic CCRCC using the GSE37989 data set. This latter analysis showed that, at the miRNA level, CCPRCC more closely resembled primary CCRCC compared with metastatic CCRCC (Fig. 2B). Table 3 shows selected differentially expressed miRNAs in 1 or more of the 3 histotypes from the cross-platform comparisons.

\subsection{Technical and independent set validation of CCPRCC microarray findings}

Among the most differentially expressed miRNAs in the microarray experiments, miR-122, miR-135a, and miR-204 were used for technical validation using qRT-PCR. The consistency between microarray data and qRT-PCR data were 4/4 (100\%) for all 3 miRNAs. In an independent set of 9 paired tumor and normal samples, we found similar trends as of our microarray data. In 2 additional CCPRCC tumor samples lacking available corresponding normal tissues, we normalized the expression of each of the above 3 miRNAs by the median of all 13 normal samples. The expression of all the 3 miRNAs in these 2 tumors showed 
similar trends with paired tumors-normal samples. Fig. 3 and Table 4 summarize our findings in technical, independent, and combined set of samples.

\section{Discussion}

We assessed the miRNA expression profile in a cohort of CCPRCC and compared our findings with miRNA expression data available in 2 public data sets from primary and metastatic CCRCC and PRCC.

Among the most differentially expressed miRNA in CCPRCC, we found miR-210, miR-122, miR-34a, miR-21, miR-34b*, and miR-489 to be up-regulated, whereas miR-4284, miR-1202, miR-135a, miR-1973, and miR-204 were down-regulated compared with normal renal parenchyma. Our microarray findings were further validated by qRT-PCR analysis in 3 of the most differentially expressed miRNAs. Our study also revealed that the miRNA expression profile of CCPRCC more closely resembled CCRCC for up-regulated miRNAs and PRCC for down-regulated miRNAs. In addition, as expected, the CCPRCC miRNA profile more closely resembled primary than metastatic CCRCC.

The finding of shared up-regulated miRNAs among CCPRCC and CCRCC could reflect an overlap in their underlying oncogenic mechanisms. One such potential mechanism is the hypoxia pathway, which ultimately leads to the expression of hypoxia-inducible factor (HIF)-related markers CAIX, glucose transporter 1, and HIF-1 $a$. In CCRCC, the hypoxia pathway is activated as a result of $V H L$ loss of function [22]. Although HIF pathway has been also found to be activated in CCPRCC [12], its activation is independent of VHL loss and due to other genetic or epigenetic factors. Several studies have documented miR-210 to be overexpressed in CCRCC [4]. miR-210 is a well-known target of HIF and a central player in the hypoxia pathway [23]. A recent report also demonstrated that miR-210 expression positively modulates HIF and correlates with CAIX expression [24]. Intriguingly, our finding of miR-210 up-regulation in CCPRCC could be implicating miR-210 in the activation of the hypoxia pathway in the absence of VHL. Evidently, such hypothesis would require verification.

Among other miRNAs that we found up-regulated in our CCPRCC tumors is miR-122. The latter has also been consistently reported to be overexpressed in CCRCC and has been proposed as a potential regulator of $V H L$ gene $[25,26]$. A recent study revealed higher miR-122 levels in primary compared with metastatic CCRCC [21]. However, its role in CCRCC pathogenesis remains to be fully determined.

miR-34a has been previously shown to be up-regulated in CCRCC. Using CCRCC cell lines, Yamamura et al [27] demonstrated that miR-34a suppresses $c-M Y C$ and its complexes and inhibits cell invasion, thus suggesting a role as a tumor suppressor in renal cancers. The pathogenic significance, if any, of our observed miR-34a up-regulation in CCPRCC should be further explored.

miR-18a was among miRNAs that we found to be up-regulated in CCPRCC and CCRCC in comparison with PRCC. The overexpression of miR-18a has previously been also reported in renal cancer [28]. It is implicated in negative feedback regulation of estrogen receptor $a$ 
(ER $a$ ) in breast cancer [29]. ER $a$ has been recently demonstrated to be a proteasomal degradation target of the VHL protein in renal carcinoma cell lines [30]. In VHL-deficient conditions, like CCRCC, ER $a$ is not sequestered and induces cell proliferation, inducing the expression of miR-18a. Our finding of up-regulated miR-18a in CCPRCC is intriguing, as other mechanisms might be responsible for its expression. Moreover, Brannon et al [31] in a meta-analysis of CCRCC gene expression databases identified a subgroup of tumors characterized by wild-type $V H L$ that morphologically could be classified as CCPRCC [11]. Interestingly, these tumors were characterized by underexpression of angiogenesis-related genes and overexpression of genes related to ER $a$. The role of the latter pathway in the pathogenesis of CCPRCC along with its interplay with miRNA merits further investigation.

We found a general down-regulation of miRNAs in CCRCC compared with CCPRCC. This is in line with the previously shown stepwise down-regulation of miRNA expression from normal to primary and metastatic tumors in renal cancer [21]. Among the miRNAs that we found to be consistently down-regulated in primary and metastatic CCRCC are members of the miR-200 family that regulate epithelial-mesenchymal transition during tumor progression [32]. miR-141 and miR-200c have been consistently shown to be downregulated in CCRCC leading to overexpression of their vascular endothelial growth factor A (VEGFA) target $[19,33]$. Although we also found the latter 2 miRNAs to be down-regulated in CCRCC, this was not true in our CCPRCC tumors. Whether the lack of down-regulation of such key miRNA, involved in the incremental progression of malignant biologic behavior of CCRCC, may be in part related to the relatively indolent behavior of CCPRCC would require further investigation.

We found miR-135a and miR-204 to be down-regulated in CCPRCC. miR-135a has been recently reported to act as a tumor suppressor in RCC cell lines by targeting c-MYC [20]. Mikhaylova et al [34] recently showed that miR-204 is a $V H L$-regulated tumor suppressor acting by inhibiting macroautophagy, with LC3B as a direct and functional target in CCRCC specimens. The mechanisms underlying miR-204 down-regulation in CCPRCC merit further investigation, specifically in regards to the function of $V H L$.

Finally, our finding that CCPRCC is more similar to PRCC than CCRCC in regards to the pattern of down-regulated miRNAs implies that the 2 types of tumors share a relatively greater number of dysregulated oncosuppressor miRNAs with PRCC than with CCRCC. The relevance of the latter finding needs to be further pursued.

A strength of our study is the performance of technical and independent set validation of our microarray findings. qRT-PCR for miR-122, miR-135a, and miR-204 found consistent results between both techniques. The 3 miRNAs showed the same trends as in the microarray experiments.

A limitation of the current study is our inability to perform functional studies due to the fact that cell lines for CCPRCC are not available. In addition, a relatively low number of samples were used. It must be noted, however, that CCPRCC are rare tumors.

In summary, we presented the miRNA expression profile of CCPRCC and compared it with the 2 other histotypes CCRCC and PRCC. Although we found some overlap in miRNA 
expression with CCRCC and PRCC, most miRNAs in CCPRCC showed a unique pattern of expression compared with CCRCC and PRCC, thus further supporting the notion that CCPRCC is a separate entity. These findings are in line with what has been reported in a very recent report [35]. Our results might help in understanding the molecular underpinning of this type of tumors.

\section{Supplementary Material}

Refer to Web version on PubMed Central for supplementary material.

\section{References}

1. Bartel DP. MicroRNAs: genomics, biogenesis, mechanism, and function. Cell. 2004; 116:281-97. [PubMed: 14744438]

2. Youssef YM, White NM, Grigull J, et al. Accurate molecular classification of kidney cancer subtypes using microRNA signature. Eur Urol. 2011; 59:721-30. [PubMed: 21272993]

3. Powers MP, Alvarez K, Kim HJ, Monzon FA. Molecular classification of adult renal epithelial neoplasms using microRNA expression and virtual karyotyping. Diagn Mol Pathol. 2011; 20:63-70. [PubMed: 21532496]

4. Slaby O, Redova M, Poprach A, et al. Identification of microRNAs associated with early relapse after nephrectomy in renal cell carcinoma patients. Genes Chromosomes Cancer. 2012; 51:707-16. [PubMed: 22492545]

5. Eble, JN.; Sauter, G.; Epstein, JI.; Sesterhenn, IA. WHO classification of tumors. Lyon, France: IARC Press; 2004. Pathology and genetics: tumors of the urinary system and male genital organs.

6. Kovacs G, Erlandsson R, Boldog F, et al. Consistent chromosome 3p deletion and loss of heterozygosity in renal cell carcinoma. Proc Natl Acad Sci U S A. 1988; 85:1571-5. [PubMed: 2894030]

7. Gayrard N, Cacheux V, Iborra F, Mourad G, Argiles A. Cytogenetic studies of 24 renal epithelial tumors with von Hippel-Lindau and fragile histidine triad protein expression correlation. Arch Pathol Lab Med. 2008; 132:965-73. [PubMed: 18517280]

8. Brunelli M, Eccher A, Gobbo S, et al. Loss of chromosome $9 \mathrm{p}$ is an independent prognostic factor in patients with clear cell renal cell carcinoma. Mod Pathol. 2008; 21:1-6. [PubMed: 17906617]

9. Tickoo SK, dePeralta-Venturina MN, Harik LR, et al. Spectrum of epithelial neoplasms in end-stage renal disease: an experience from 66 tumor-bearing kidneys with emphasis on histologic patterns distinct from those in sporadic adult renal neoplasia. Am J Surg Pathol. 2006; 30:141-53. [PubMed: 16434887]

10. Gobbo S, Eble JN, Grignon DJ, et al. Clear cell papillary renal cell carcinoma: a distinct histopathologic and molecular genetic entity. Am J Surg Pathol. 2008; 32:1239-45. [PubMed: 18594469]

11. Williamson SR, Eble JN, Cheng L, Grignon DJ. Clear cell papillary renal cell carcinoma: differential diagnosis and extended immunohistochemical profile. Mod Pathol. 2013; 26:697-708. [PubMed: 23238627]

12. Rohan SM, Xiao Y, Liang Y, et al. Clear-cell papillary renal cell carcinoma: molecular and immunohistochemical analysis with emphasis on the von Hippel-Lindau gene and hypoxiainducible factor pathway-related proteins. Mod Pathol. 2011; 24:1207-20. [PubMed: 21602815]

13. Martignoni G, Brunelli M, Segala D, et al. VHL mutation, VHL methylation, chromosome $3 p$ and whole genomic status in clear cell papillary renal cell carcinoma. Mod Pathol. 2013; 26:233A.

14. Zhan H, Cardozo C, Yu W, et al. MicroRNA deregulation in polycythemia vera and essential thrombocythemia patients. Blood Cells Mol Dis. 2013; 50:190-5. [PubMed: 23265742]

15. Livak KJ, Schmittgen TD. Analysis of relative gene expression data using real-time quantitative PCR and the 2(-Delta Delta C(T)) Method. Methods. 2001; 25:402-8. [PubMed: 11846609] 
16. Benassi B, Flavin R, Marchionni L, et al. MYC is activated by USP2a-mediated modulation of microRNAs in prostate cancer. Cancer Discov. 2012; 2:236-47. [PubMed: 22585994]

17. Schaeffer EM, Marchionni L, Huang Z, et al. Androgen-induced programs for prostate epithelial growth and invasion arise in embryogenesis and are reactivated in cancer. Oncogene. 2008; 27:7180-91. [PubMed: 18794802]

18. Ross AE, Marchionni L, Vuica-Ross M, et al. Gene expression pathways of high grade localized prostate cancer. Prostate. 2011; 71:1568-78. [PubMed: 21360566]

19. Nakada C, Matsuura K, Tsukamoto Y, et al. Genome-wide microRNA expression profiling in renal cell carcinoma: significant down-regulation of miR-141 and miR-200c. J Pathol. 2008; 216:41827. [PubMed: 18925646]

20. Yamada Y, Hidaka H, Seki N, et al. Tumor-suppressive microRNA-135a inhibits cancer cell proliferation by targeting the c-MYC oncogene in renal cell carcinoma. Cancer Sci. 2013; 104:304-12. [PubMed: 23176581]

21. Wotschofsky Z, Liep J, Meyer HA, et al. Identification of metastamirs as metastasis-associated microRNAs in clear cell renal cell carcinomas. Int J Biol Sci. 2012; 8:1363-74. [PubMed: 23139634]

22. Wiesener MS, Munchenhagen PM, Berger I, et al. Constitutive activation of hypoxia-inducible genes related to overexpression of hypoxia-inducible factor-1alpha in clear cell renal carcinomas. Cancer Res. 2001; 61:5215-22. [PubMed: 11431362]

23. Chan SY, Loscalzo J. MicroRNA-210: a unique and pleiotropic hypoxamir. Cell Cycle. 2010; 9:1072-83. [PubMed: 20237418]

24. Chang W, Lee CY, Park JH, et al. Survival of hypoxic human mesenchymal stem cells is enhanced by a positive feedback loop involving miR-210 and hypoxia-inducible factor 1. J Vet Sci. 2013; 14:69-76. [PubMed: 23388440]

25. White NM, Bao TT, Grigull J, et al. miRNA profiling for clear cell renal cell carcinoma: biomarker discovery and identification of potential controls and consequences of miRNA dysregulation. $\mathbf{J}$ Urol. 2011; 186:1077-83. [PubMed: 21784468]

26. Zhou L, Chen J, Li Z, et al. Integrated profiling of microRNAs and mRNAs: microRNAs located on Xq27. 3 associate with clear cell renal cell carcinoma. PLoS One. 2010; 5:e15224. [PubMed: 21253009]

27. Yamamura S, Saini S, Majid S, et al. MicroRNA-34a suppresses malignant transformation by targeting c-Myc transcriptional complexes in human renal cell carcinoma. Carcinogenesis. 2012; 33:294-300. [PubMed: 22159222]

28. Neal CS, Michael MZ, Rawlings LH, Van der Hoek MB, Gleadle JM. The VHL-dependent regulation of microRNAs in renal cancer. BMC Med. 2010; 8 [64,7015-8-64].

29. Castellano L, Giamas G, Jacob J, et al. The estrogen receptor-alpha-induced microRNA signature regulates itself and its transcriptional response. Proc Natl Acad Sci U S A. 2009; 106:15732-7. [PubMed: 19706389]

30. Jung M, Mollenkopf HJ, Grimm C, et al. MicroRNA profiling of clear cell renal cell cancer identifies a robust signature to define renal malignancy. J Cell Mol Med. 2009; 13:3918-28. [PubMed: 19228262]

31. Brannon AR, Haake SM, Hacker KE, et al. Meta-analysis of clear cell renal cell carcinoma gene expression defines a variant subgroup and identifies gender influences on tumor biology. Eur Urol. 2012; 61:258-68. [PubMed: 22030119]

32. Davalos V, Moutinho C, Villanueva A, et al. Dynamic epigenetic regulation of the microRNA-200 family mediates epithelial and mesenchymal transitions in human tumorigenesis. Oncogene. 2012; 31:2062-74. [PubMed: 21874049]

33. Liu H, Brannon AR, Reddy AR, et al. Identifying mRNA targets of microRNA dysregulated in cancer: with application to clear cell renal cell carcinoma. BMC Syst Biol. 2010; 4 [51,0509-4-51].

34. Mikhaylova O, Stratton Y, Hall D, et al. VHL-regulated MiR-204 suppresses tumor growth through inhibition of LC3B-mediated autophagy in renal clear cell carcinoma. Cancer Cell. 2012; 21:532-46. [PubMed: 22516261] 
35. Lawrie $\mathrm{CH}$, Larrea E, Larrinaga G, et al. Targeted next generation sequencing and non-coding RNA expression analysis of clear cell papillary renal cell carcinoma suggests distinct pathological mechanisms from other renal tumour subtypes. J Pathol. 2014; 232:32-42. [PubMed: 24155122] 

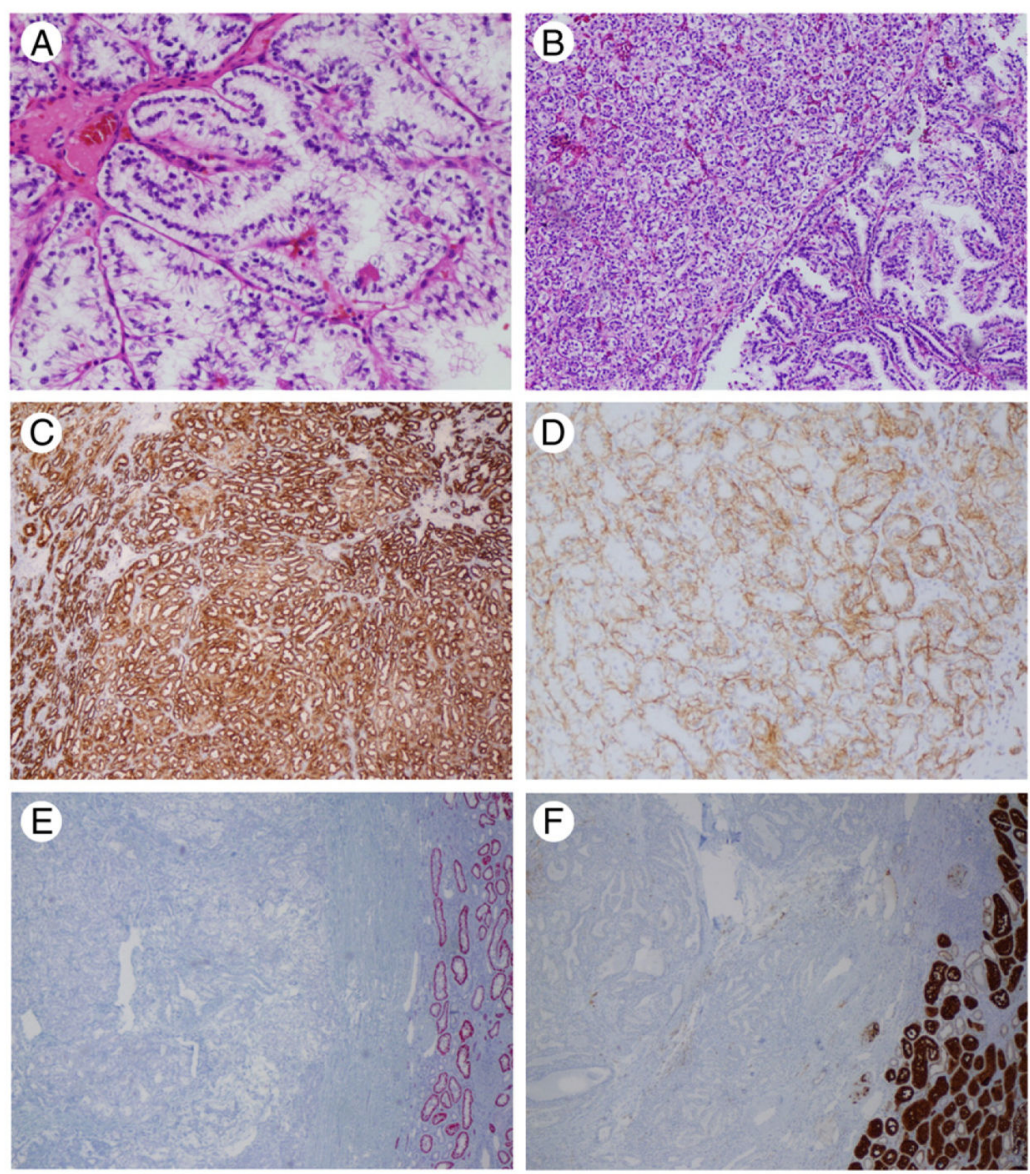

Fig. 1.

Histologic and immunohistochemical features of CCPRCC. A, Tumor cells showed typical nuclear arrangement away from the basement membrane. B, Small tubules and acini imparting a solid appearance admixed with delicate papillary structures. C, CK7 showed strong and diffuse positivity. D, CAIX showed the typical basal staining lacking the luminal aspect. E, AMACR resulted negative in all cases; normal distal tubules were positive. F, CD10 was negative or weakly positive. A, Original magnification $\mathrm{HE}, \times 20$; $\mathrm{B}, \mathrm{HE}, \times 10$; C to $\mathrm{F}, \times 10$. 

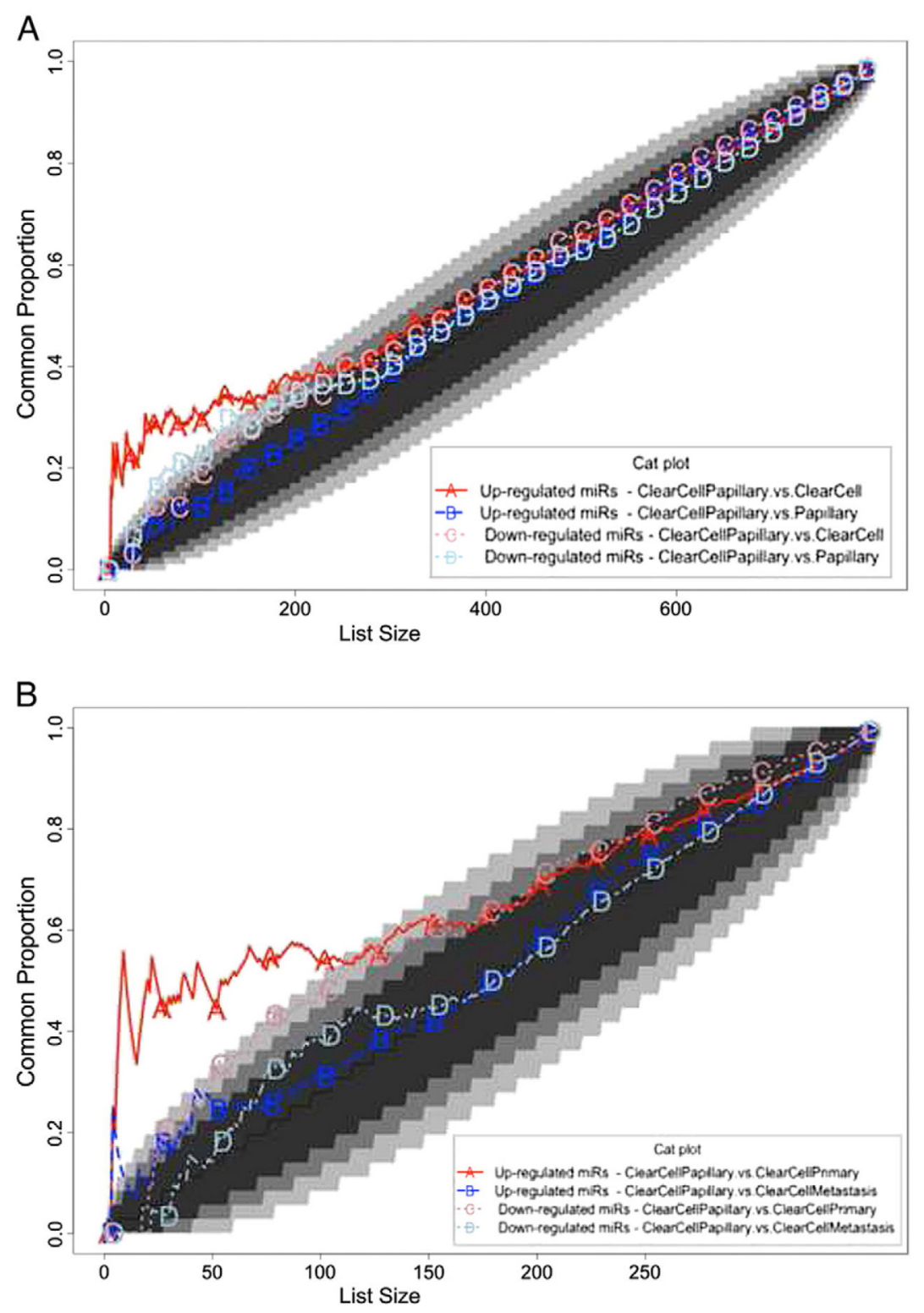

Fig. 2.

Consensus among miRNA profiles associated with distinct renal carcinoma histotypes and stages. Lines above the $45^{\circ}$ diagonal lines correspond to comparisons having more agreement than would be expected by chance. Lines in the white area of the graph have a probability of agreeing by chance of less than $1 \mathrm{E}-06$. Genes were ranked by t-statistic as obtained from the linear model analysis. A, CAT curves for the following comparisons: miRNA up-regulated and down-regulated in clear cell papillary and clear cell tumors; miRNA up-regulated and down-regulated in papillary and clear cell papillary tumors. B, CAT curves for the following comparisons: miRNA up-regulated and down-regulated in clear cell papillary and in primary clear cell tumors; miRNA up-regulated and downregulated in clear cell papillary and clear cells tumor metastasis. 

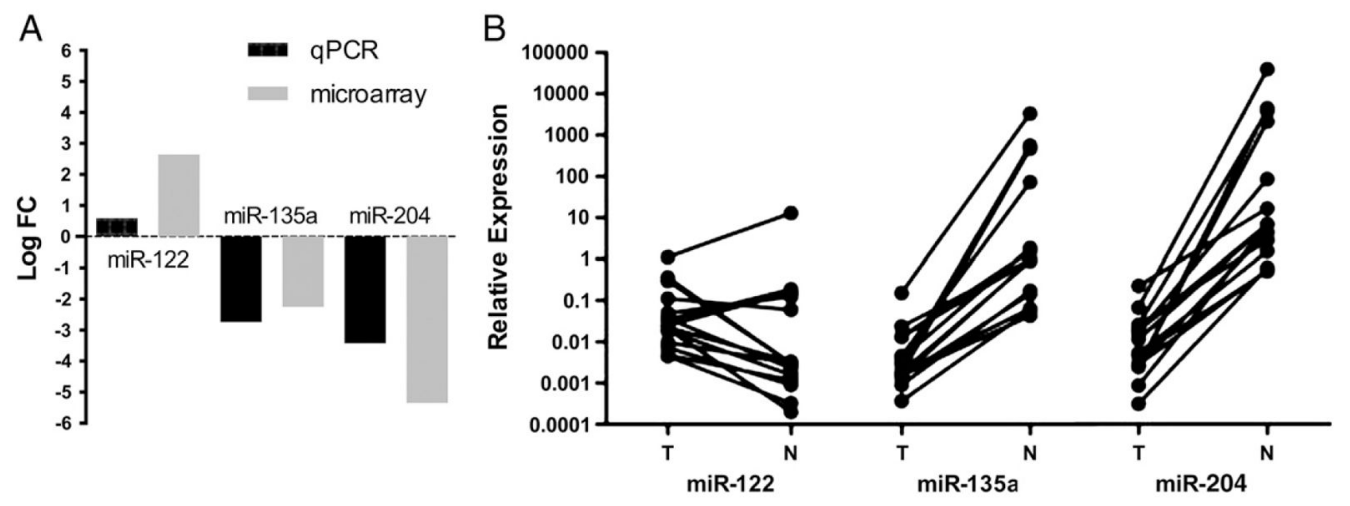

Fig. 3.

Technical and independent set validation of miR-122, miR-135a, and miR-204. A, Average fold changes of samples used for the microarray experiments (4) and qRT-PCR experiments (11). B, Relative expression on the 3 miRNAs in all 15 cases. 


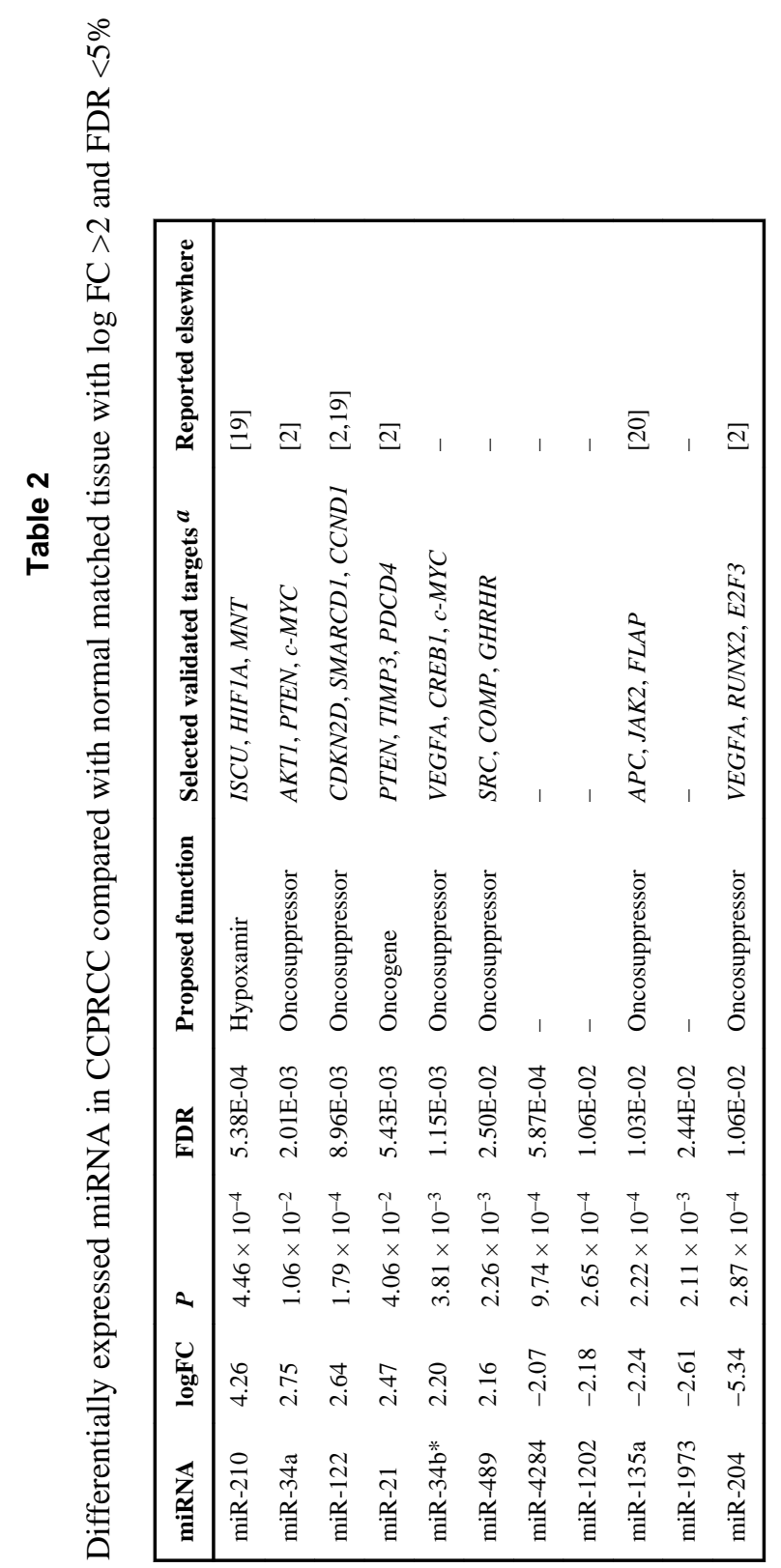

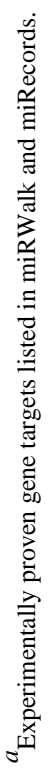

Hum Pathol. Author manuscript; available in PMC 2015 February 18. 
Table 3

Selected differentially expressed miRNAs in CCPRCC, CCRCC, and PRCC

\begin{tabular}{|lllll|}
\hline miRNA & CCPRCC & CCRCC & PRCC & Selected validated targets \\
\hline miR-210 & Up & Up & NS & EFNA, MNT, VMP1 \\
miR-155 & Up & Up & NS & AGTR1, BACH1, LDOC1 \\
miR106b & Up & Up & NS & E2F1, VEGFA, CDKN1A \\
miR-15a & Up & Up & NS & BCL2, VEGFA, PDCD4 \\
miR-18a & Up & Up & NS & BIM, CTGF, ESR1 \\
miR-130a & Up & NS & NS & - \\
miR135b & Up & NS & NS & APC \\
miR-101 & Up & NS & NS & - \\
miR-10b & NS & Down & Down & HOXD10, KLF4, SFRS1 \\
miR-141 & NS & Down & - & CLOCK, SERBP1, ELMO2 \\
miR-200a & NS & Down & Down & ZEB1, ZEB2, ELMO2 \\
miR-200b & NS & Down & Down & ZEB1, ZEB2, ELMO2 \\
miR-200c & NS & Down & Down & ZEB1, ERRFI1, JAG1 \\
miR-26a & NS & Down & Down & SMAD1, PLAG1, TGFBR2 \\
miR-218 & NS & Down & Down & LAMB3, COL1A1, ECOP \\
miR-187 & NS & Down & Down & - \\
miR-138 & Down & Down & Down & RHOC, KRT, ROCK2 \\
miR-422a & Down & NS & Down & - \\
miR-648 & Down & NS & NS & - \\
miR-215 & Down & NS & NS & DHFR, TYMS, DTL, WNK1 \\
miR-663 & Down & NS & NS & - \\
miR-204 & Down & Down & NS & ARPC1B, MMP3, MMP9 \\
miR-135a & Down & Down & NS & APC, FLAP, JAK2 \\
\hline & & & & \\
\hline
\end{tabular}

Abbreviations: CCPRCC, clear cell papillary renal cell carcinoma; CCRCC, clear cell renal cell carcinoma; PRCC, papillary renal cell carcinoma; * experimentally proven gene targets listed in miRWalk and miRecords; NS, not significantly different compared with matched normal.

${ }^{a}$ Experimentally proven gene targets listed in miRWalk and miRecords. 


\section{Table 4}

Summary of qRT-PCR results for technical and independent set validation

\begin{tabular}{|lcrr|}
\hline & miR-122 Up & miR-135 Down & miR-204 Down \\
\hline TV & $4 / 4(100 \%)$ & $4 / 4(100 \%)$ & $4 / 4(100 \%)$ \\
ISV & $7 / 11(63 \%)$ & $11 / 11(100 \%)$ & $11 / 11(100 \%)$ \\
TV + ISV & $11 / 15(73 \%)$ & $15 / 15(100 \%)$ & $15 / 15(100 \%)$ \\
\hline
\end{tabular}

Abbreviations: TV, technical validation; ISV, independent set validation. 